\title{
Impact de la nutrition sur la morbimortalité en réanimation*
}

\section{Impact of nutritional support on morbidity and mortality in critical illness}

\author{
M.M. Berger \\ Reçu le 9 octobre 2011 ; accepté le 3 novembre 2011 \\ (C) SRLF et Springer-Verlag France 2011
}

Résumé Les patients admis en réanimation sont particulièrement fragiles et sensibles à la fois aux effets de la dénutrition et de la suralimentation qui provoquent infections, ventilation prolongée et surmortalité. La définition précise de besoins énergétiques devient d'autant plus importante : la calorimétrie indirecte reste à ce jour le seul outil fiable, bien qu'elle ne soit pas applicable à tout patient. Pour citer cette revue : Réanimation 21 (2012).

Mots clés malnutrition $\cdot$ suralimentation $\cdot$ déficit énergétique $\cdot$ calorimétrie $\cdot$ infection

\begin{abstract}
Critically ill patients are particularly fragile and sensitive both to under- and overfeeding, which cause infections, prolonged ventilation and overmortality. The exact definition of energy requirements is therefore extremely important; indirect calorimetry remains the only reliable tool in the clinical setting; however, it is not always applicable.
\end{abstract}

\section{To cite this journal: Réanimation 21 (2012).}

Keywords Underfeeding · Overfeeding · Energy deficit · Calorimetry $\cdot$ Infection

\section{Introduction}

La patientèle admise en réanimation a beaucoup évolué au cours des deux dernières décennies. Les patients traités sont en moyenne nettement plus âgés, souffrent de nombreuses comorbidités et sont particulièrement sensibles aux effets de la dénutrition. Ces patients sont aujourd'hui éligibles pour des chirurgies lourdes programmées ou réa-

M.M. Berger $(\bowtie)$

Service de médecine intensive adulte et brûlés,

CHU Vaudois, BH 08.612, 46, rue du Bugnon,

1011-Lausanne, Suisse

e-mail : mette.berger@chuv.ch

\footnotetext{
* Cet article correspond à la conférence faite par l'auteur au congrès de la SRLF 2012 dans la session : Assistance nutritionnelle en réanimation.
}

lisées en urgence, inimaginables encore il y a 20 ans. Les traitements de réanimation ont aussi changé. Les séjours des patients se sont prolongés dans une proportion importante de cas : alors que les affections aiguës ( $<1$ semaine) prédominaient dans les années 1990, on observe une augmentation des séjours «aigus prolongés » (deux-six semaines), avec l'apparition de situations " aiguës chroniques » (de six semaines à plusieurs mois) [1]. Face à cette évolution, il est aisément concevable que les besoins nutritionnels évoluent au cours du temps en particulier avec la fonte de la masse maigre et l'inactivité liée à l'alitement prolongé [2].

Par ailleurs, la malnutrition hospitalière persiste malgré son diagnostic et des directives édictées au niveau du Conseil de l'Europe [3]. Les patients sont dénutris à l'hôpital dans près de $50 \%$ des cas comme le montre une étude qui a porté sur une cohorte de 1707 patients hospitalisés [4] : les besoins en énergie et protéines, basés sur les recommandations de l'European Society of Clinical Nutrition \& Metabolism (ESPEN), ne sont couverts que dans moins d'un quart des patients $(24,4 \%)$ [5]. La dénutrition se produit soit par insuffisance d'apports ou à cause de l'augmentation des besoins, non satisfaits par les apports « standard». Les patients de réanimation y sont particulièrement exposés, en outre à cause de leur vulnérabilité provoquée par l'affection aiguë et la réponse inflammatoire, mais aussi en raison des caractéristiques exposées plus haut. Les travaux rapportant l'impact négatif de la malnutrition aiguë en soins intensifs sont nombreux. Le Tableau 1 résume les observations de quelques études et des complications observées liées à la nutrition.

\section{Définitions des besoins énergétiques}

Cet objectif apparemment simple est devenu l'un des plus controversés, et cela malgré l'existence de guidelines qui recommandent de prescrire $20-25 \mathrm{kcal} / \mathrm{kg}$ par jour durant les premiers jours puis d'augmenter progressivement à 25-30 kcal/kg par jour, selon que le patient souffre d'une 
Tableau 1 Études caractérisées par de la sous- ou suralimentation en réanimation — liste non exhaustive

\begin{tabular}{|ll|}
\hline Sous-alimentation & $\begin{array}{l}\text { Nombre de patients, type } \\
\text { (médical, chirurgical, mixte) } \\
\text { et détails des résultats }\end{array}$ \\
\hline $\begin{array}{l}\text { Bartlett et al., } \\
1982[18]\end{array}$ & $\begin{array}{l}n=57, \text { chirurgical, NE et NP } \\
\text { Bilan négatif associé à une } \uparrow \text { défaillances } \\
\text { d'organe (respiratoire, hépatique, rénal, } \\
\text { cardiaque) et une } \uparrow \text { des infections }\end{array}$
\end{tabular}

Atkinson et al., $1998[25]$

Rubinson, et al., $2004[20]$

Villet et al., 2005 [11]

Dvir et al., 2005 [12] $n=277$ Étude d'immunonutrition entérale

Apport moyen de $14 \mathrm{kcal} / \mathrm{kg}$ par jour dans les 2 groupes compromettant l'analyse de données

Déficit moyen de $-1200 \mathrm{kcal} / \mathrm{j}$ associé à $\uparrow$ mortalité à 2 semaines $(p=0,01)$

$n=138$, médical

Bactériémies directement liées à l'apport

d'énergie avec une augmentation marquée chez les patients recevant moins de $25 \%$ de la cible fixée selon les recommandations de l'ACCP Différence visible dès $\mathrm{j} 7$ après admission

$n=48$, chirurgical

$\uparrow$ Complications ( $p=0,048)$,

dont les infections $(p=0,0049)$

$n=50$, mixte

$\uparrow$ Insuffisance respiratoire $(p=0,0003)$, sepsis $(p=0,0035)$, insuffisance rénale ( $p=0,0001)$, ulcères de décubitus

$(p=0,013)$, besoins en chirurgie

$(p=0,023)$, complications totales

$(p=0,0001)$
Suralimentation Nombre de patients, type (médical, chirurgical, mixte) et détails des résultats

Herndon et al., $\quad n=39$, brûlés, NE versus nutrition 1989 [24] combinée

Hyperalimentation dans les 2 groupes : cible selon Curreri, largement atteinte dans le groupe combinée $\uparrow$ Mortalité dans le groupe nutrition combinée (63 versus $26 \%$ ) avec dans les 2 groupes : diminution de l'immunité, hépatomégalie et cholestase

Battistella et al., $n=57$, polytraumatisés, NP dès j4, 1997 [26] avec ou sans lipides. Cible : $30 \mathrm{kcal} / \mathrm{kg}$ non protéique. Les $25 \%$ de lipides dans le groupe lipides n'étaient pas compensés dans le groupe « sans » $(23 \mathrm{kcal} / \mathrm{kg})$ :

$+1,5 \mathrm{~g} / \mathrm{kg}$ de protéines $(6 \mathrm{kcal} / \mathrm{kg})$ dans les 2 groupes. Le groupe lipides était donc hyperalimenté avec $\uparrow$ infections, jours de ventilation, durée de séjour en réanimation et à l'hôpital

Ahrens et al., $\quad n=40$, chirurgical

2005 [27] Randomisation en NP « hypo » (20 kcal/kg par jour non protéique) ou NP standard (30 kcal/kg par jour non protéique) : auxquelles s'ajoutaient des émulsions lipidiques 3 par semaine, soit $3 \times 1000=3000 \mathrm{kcal}$ à tous les patients

Pas d'amélioration du contrôle glycémique

Dissanaike et al., $n=200$, mixte, NP

2007 [28] Des apports $>26 \mathrm{kcal} / \mathrm{kg}$ par jour étaient associés à des bactériémies dans le groupe bactériémique, l'apport énergétique moyen était de $35 \mathrm{kcal} / \mathrm{kg}$; " iso » était de fait hypercalorique

Arabi et al., $\quad n=523$, mixte (sous-étude d'une étude 2010 [13] de contrôle portant sur l'insuline) Cible d'énergie fixée par l'équation de Harris \& Benedict avec facteur-stress de « correction » variable Patients classifiés en « tertiles d'atteinte de la cible » : les patients avec la meilleure couverture de la cible avaient significativement plus

(Suite page suivante) 


\begin{tabular}{|c|c|c|c|}
\hline Sous-alimentation & $\begin{array}{l}\text { Nombre de patients, type } \\
\text { (médical, chirurgical, mixte) } \\
\text { et détails des résultats }\end{array}$ & Suralimentation & $\begin{array}{l}\text { Nombre de patients, type } \\
\text { (médical, chirurgical, mixte) } \\
\text { et détails des résultats }\end{array}$ \\
\hline $\begin{array}{l}\text { Badjatia et al., } \\
2010 \text { [29] }\end{array}$ & $\begin{array}{l}n=58 \text {, neurochirurgie } \\
\uparrow \text { Complications infectieuses après } \\
\text { hémorragie sous-arachnoïdienne } \\
\text { avec un bilan énergétique moyen } \\
\text { au } 7^{\text {e }} \text { jour de }-117 \mathrm{kcal} / \mathrm{kg}\end{array}$ & $\begin{array}{l}\text { Singer et al., } \\
2010[14]- \\
\text { TICACOS }\end{array}$ & $\begin{array}{l}\text { de pneumonies et une mortalité ajustée } \\
\text { plus élevée } \\
n=102 \text {, mixte } \\
\text { Cibles d'énergie fixées par calorimétrie } \\
\text { (toutes les } 48 \mathrm{~h} \text { ) ou par l'équation } \\
25 \mathrm{kcal} / \mathrm{kg} \text {. Le groupe calorimétrie } \\
\text { ne tenait pas compte des calories non } \\
\text { nutritionnelles pour adapter sa cible } \\
\text { résultant en une modeste surnutrition } \\
\text { systématique Tendance à } \downarrow \text { mortalité } \\
\text { dans le groupe calorimétrie malgré, } \\
\uparrow \text { infections et durée de ventilation } \\
\text { mécanique }\end{array}$ \\
\hline $\begin{array}{l}\text { Alberda et al., } \\
2009 \text { [30] }\end{array}$ & $\begin{array}{l}n=2772, \text { mixte, patients ventilés } \\
\text { Apport énergétique moyen de } 14 \mathrm{kcal} / \mathrm{kg} \\
\text { par jour, } 1034 \mathrm{kcal} / \mathrm{j} \text { et } 47 \mathrm{~g} \text { de protéine } / \mathrm{j} \\
\uparrow 100 \mathrm{kcal} / \mathrm{j} \text { associée à une } \downarrow \text { mortalité } \\
(p=0,014) \text { et } \uparrow \text { jours sans ventilation } \\
\text { mécanique }(p=0,003)\end{array}$ & $\begin{array}{l}\text { Casaer et al., } \\
2011[15]- \\
\text { EPaNIC }\end{array}$ & $\begin{array}{l}n=4640 \\
\text { NP dès } \mathrm{j} 1 \text { versus dès } \mathrm{j} 7 \text { chez des patients } \\
\text { dont } 60 \% \text { étaient des cas de chirurgie } \\
\text { cardiaque. } \\
\text { NE débutée des } \mathrm{j} 2-\mathrm{j} 3 \text { et augmentée très } \\
\text { lentement Cibles d'énergie fixées } \\
\text { par des équations sans vérification } \\
\text { calorimétrique et aboutissant } \\
\text { à une suralimentation précoce } \\
\text { chez au moins } 50 \% \text { des patients } \\
\uparrow \text { Complications infectieuses } \\
\text { ( } p=0,008) \text {, cholestase }(p<0,001) \text {, } \\
\text { durée de ventilation mécanique et durée } \\
\text { de réanimation mais sans } \uparrow \text { mortalité }\end{array}$ \\
\hline $\begin{array}{l}\text { Faisy et al., } \\
2009[21]\end{array}$ & $\begin{array}{l}n=38 \text {, médical, patients ventilés } \\
\text { pendant } 7 \text { jours au moins, NE } \\
\uparrow \text { Mortalité }(p=0,003) \text { dès que le déficit } \\
\text { excède } 5021 \mathrm{~kJ} / \mathrm{j}(1220 \mathrm{kcal} / \mathrm{j})\end{array}$ & & \\
\hline $\begin{array}{l}\text { Faisy et al., } \\
2011 \text { [31] }\end{array}$ & $\begin{array}{l}n=70, \text { médical, patients ventilés, NE } \\
\uparrow \text { Pneumonies acquises sous ventilation } \\
\text { à Staphylococcus aureus chez les } \\
\text { patients avec les déficits les plus élevés }\end{array}$ & & \\
\hline
\end{tabular}

pathologie médicale ou chirurgicale [6]. La détermination d'une cible d'énergie optimale est en effet difficile chez les patients de réanimation, en raison de la variabilité du métabolisme basal induit par l'état de choc, la fièvre, la fonte de masse maigre, les interventions chirurgicales, la sédation... [7]. Les besoins varient aussi dans le temps, ce qui accroît la difficulté de définir le besoin alimentaire adéquat. L'utilisation d'équations prédictives requiert de plus la connaissance $\mathrm{du}$ poids « sec » du patient avant l'admission, souvent inconnu. Tout cela contribue à l'incertitude quant à la prescription nutritionnelle.

La sur- et sous-alimentation sont toutes deux néfastes et produisent paradoxalement des effets similaires aux extrêmes (Tableau 2) : elles aboutissent, toutes deux, à des perturbations de l'immunité avec l'apparition de complications infectieuses.

Pour cette raison, les guidelines nutritionnelles recommandent de mesurer la dépense énergétique de base (DER) 
Tableau 2 Effets de la sur- et de la sous-alimentation

\begin{tabular}{|ll|}
\hline Sous-alimentation & Suralimentation \\
\hline Infections & Hyperglycémie \\
Faiblesse musculaire $(\downarrow$ masse maigre) & Foie gras (stimulation de la lipogenèse de novo) \\
Sevrage respiratoire difficile avec prolongation de la durée & Sevrage respiratoire difficile (production élevée de $\left.\mathrm{CO}_{2}\right)$ \\
de ventilation mécanique (faiblesse musculaire) & Stress, tachycardie ( $\uparrow$ catécholamines) \\
Cicatrisation retardée & Acidose métabolique \\
Séjour prolongé (soins intensifs et hôpital) & Urémie \\
& Dépression immunitaire : infections
\end{tabular}

par calorimétrie indirecte [6]. Le principe physiologique sous-jacent est que la mesure de la consommation d' $\mathrm{O}_{2}$ $\left(\mathrm{VO}_{2}\right)$ et de la production de $\mathrm{CO}_{2}\left(\mathrm{VCO}_{2}\right)$ reflète les besoins énergétiques au niveau cellulaire. L'hypothèse sous-jacente est que dans un état stable, les échanges gazeux à l'équilibre reflètent les échanges gazeux mitochondriaux, mesurant ainsi indirectement la phosphorylation oxydative. Les besoins énergétiques sont ensuite extrapolés en utilisant l'équation de Weir [8] : énergie totale $=3,9 \times$ volume $\mathrm{O}_{2}$ consommé (1) $+1,1 \times$ volume $\mathrm{CO}_{2}$ produit (1). Il y a un certain nombre d'obstacles à cette mesure [9], qui reste malgré tout la manière la plus accessible pour déterminer les besoins énergétiques en comparaison aux autres méthodes comme la DEXA (dual-emission X-ray absorptiometry) ou à des mesures isotopiques.

\section{Quelle durée de jeûne est tolérable en réanimation sans danger?}

Le délai tolérable de jeûne reste très débattu, tout comme l'importance du déficit calorique acceptable. Les études sur volontaires sains montrent que des perturbations hépatiques et mitochondriales surviennent déjà après 12-18 heures. Comparé à l'état «nourri », 12 et 18 heures de jeûne complet provoquent une diminution respective de 29 et $57 \%$ du contenu hépatique en glycogène $(p<0,001)$. La réalimentation avec des suppléments glucidiques oraux ne corrige que partiellement cette baisse. Le jeûne court (jusqu'à 24 heures) modifie négativement la fonction mitochondriale des cellules mononucléaires et augmente la teneur en lipides intramyocellulaires au niveau des muscles du mollet [10].

L'analyse détaillée de plusieurs études prônant la nutrition hypocalorique montre que les groupes prétendument isocaloriques sont en fait suralimentés (Tableau 1). De plus, à l'exception de cinq études [11-15], les bilans d'énergie sont calculés sans inclure les calories « non nutritionnelles » qui ne peuvent être facilement comptabilisées qu'avec un système informatique, omettant ainsi les quantités parfois importantes d'énergie délivrées sous forme de glucose $5 \%$ et des lipides provenant du sédatif propofol. Tous les substrats doivent être inclus dans les calculs énergétiques [16] qui peuvent aisément représenter alors plus de $300 \mathrm{kcal} / \mathrm{j}$ [17] : comment imaginer que ces substrats ne soient pas utilisés au prétexte qu'ils n'ont pas été donnés pour des raisons nutritionnelles?

\section{À partir de quand apparaissent les conséquences de la malnutrition ?}

Plusieurs études donnent des indices temporels sur le moment où apparaissent les effets d'un déficit énergétique. La première étude à démontrer en soins intensifs un impact négatif du déficit d'énergie cumulé sur le devenir clinique a été réalisée aux États-Unis chez 75 patients [18] : avec des cibles fixées par calorimétrie, les auteurs ont montré qu'un déficit cumulé excédant -10 $000 \mathrm{kcal}$ était associé à une augmentation de la mortalité : il s'agissait là d'une étude sur le séjour complet de réanimation, les déficits d'énergie se retrouvant en réanimation chez 50 à $60 \%$ des patients $[11,12,19]$.

L'étude de Rubinson et al. a montré la survenue de complications infectieuses sous forme de bactériémies dès j7 [20]. Les études de Villet et al. [11] ainsi que de Dvir et al. [12] ont montré une augmentation des complications dès le cinquième jour. L'étude de Faisy et al. a montré une augmentation de mortalité survenant déjà à deux semaines [21].

Deux études prospectives conduites dans des services ayant des protocoles de nutrition et utilisant la calorimétrie indirecte pour valider les cibles d'énergie ainsi que le même système informatisé (Metavision, iMDsoft, Tel-Aviv) $[11,12]$ ont montré une proportionnalité entre un déficit d'énergie croissant et le nombre de complications, particulièrement infectieuses. Les deux études ont constaté la survenue de déficits chez respectivement 55 et $60 \%$ de leurs patients. Le seuil de bilan d'énergie cumulé auquel survient l'augmentation des complications se situait entre -8000 et $4000 \mathrm{kcal}$, ce qui correspondait à -110 et à $-50 \mathrm{kcal} / \mathrm{kg}$. Une étude randomisée contrôlée bicentrique suisse (SPN Trial), enrôlant 305 patients [22] et basant ses prescriptions d'énergie sur de la calorimétrie indirecte $(100 \%$ selon la mesure avec, inclusion dans les calculs des apports non nutritionnels), a montré qu'une couverture complète des besoins d'énergie dès le quatrième jour par une parentérale d'appoint 
évitant la suralimentation aboutissait à la réduction des complications infectieuses dès le neuvième jour : l'intervention débutait avec un déficit moyen cumulé de $-3900 \mathrm{kcal}$ (environ $-50 \mathrm{kcal} / \mathrm{kg}$ ), ce qui correspondait au seuil de l'étude israélienne. Il semble donc bien, malgré tout, qu'il y ait un seuil critique de déficit énergétique cumulé et un délai maximal de jeûne.

\section{Peut-on faire du rattrapage en cas de déficit ?}

Une caractéristique importante des patients de réanimation est leur intolérance à la suralimentation. Une grande étude multicentrique espagnole, conduite dans 40 unités de réanimation et incluant 725 patients sous nutrition entérale ou parentérale, a montré que la suralimentation définie comme des apports supérieurs à $27 \mathrm{kcal} / \mathrm{kg}$ était un des déterminants importants de la perturbation de la fonction hépatique [23].
Le problème semble survenir dès que l'on dépasse les besoins mesurés d'environ $20 \%$, avec une élévation précoce dès le troisième-quatrième jour des transaminases (cytolyse hépatique), suivie de l'apparition d'une cholestase et/ou d'une combinaison cytolyse/cholestase [23].

La conséquence est qu'un éventuel déficit énergétique accumulé en début de séjour ne peut pas être rattrapé par une suralimentation systématique après. Le " gavage » provoque la stéatose hépatique très rapidement, le « foie gras » qui n'est bénéfique en termes culinaires que chez les oies et les canards.

Dans le cas d'un déficit d'énergie par insuffisance d'apports, les patients encourent une «double peine»: d'abord, ils risquent les complications de la dénutrition, et ensuite celles de la surnutrition. La meilleure stratégie va donc consister en la prévention de la création d'un déficit d'énergie précoce.

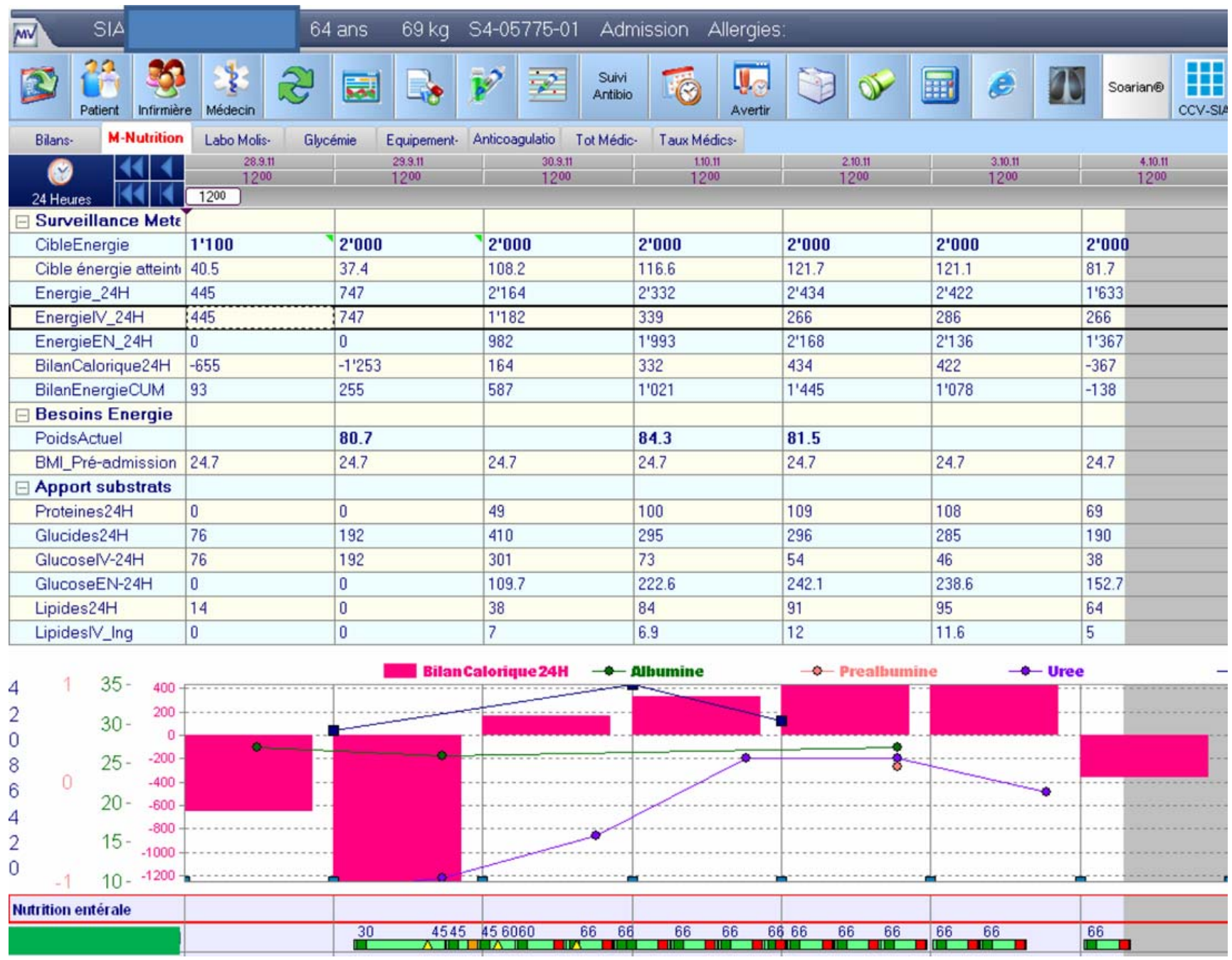

Fig. 1 Écran de suivi nutritionnel montrant la composante non nutritionnelle des apports énergétiques lors de nutrition exclusivement entérale : les lignes « Energie IV » (surlignée sur la figure), décomposée en « glucose IV » et « lipides IV » donnent l'origine de cette énergie « non prescrite » qui peut être très importante. Chaque colonne représente 24 heures 


\section{Conclusion}

La nutrition a un impact certain sur la morbimortalité des patients de réanimation. Cela est vrai que le patient se trouve sur versant hypo- ou hyper- de la couverture de ses besoins nutritionnels : le patient de réanimation est très sensible au deux. Plusieurs études à méthodologie incomplète ont augmenté la confusion sur les objectifs de la «nutrithérapie» : la confusion vient en grande partie de calculs de bilan et d'apports incomplets, faisant passer la suralimentation pour de la nutrition isocalorique. Il s'agit donc d'utiliser des cibles raisonnables, d'utiliser la nutrition entérale précoce comme le proposent les guidelines et d'éviter de dépasser des bilans cumulés négatifs excédant les -60 à $-70 \mathrm{kcal} / \mathrm{kg}$. Cette approche prudente est associée à des résultats cliniques positifs et à une prévention des infections (Fig. 1).

Conflit d'intérêt : Research supported by Grants: Aguettant, Baxter, BBraun, Fresenius Kabi. Lecturer fro: Baxter, BBraun, Fresenius Kabi, Nestlé-Novartis. Advisory board: Baxter, Aguettant.

\section{Références}

1. Nelson JE, Cox CE, Hope AA, Carson SS (2010) Chronic critical illness. Am J Respir Crit Care Med 182:446-54

2. Biolo G, Ciocchi B, Stulle M, et al (2007) Calorie restriction accelerates the catabolism of lean body mass during $2 \mathrm{wk}$ of bed rest. Am J Clin Nutr 86:366-72

3. Beck AM, Balknäs UN, Fürst P, et al (2001) Food and nutritional care in hospitals: how to prevent undernutrition - report and guidelines from the Council of Europe. Clin Nutr 20:455-60

4. Thibault R, Chikhi M, Clerc A, et al (2011) Assessment of food intake in hospitalised patients: a 10-year comparative study of a prospective hospital survey. Clin Nutr 30:289-96

5. Dupertuis YM, Kossovsky MP, Kyle UG, et al (2003) Food intake in 1,707 hospitalised patients: a prospective comprehensive hospital survey. Clin Nutr 22:115-23

6. Singer P, Berger MM, Van den Berghe G, et al (2009) ESPEN guidelines on parenteral nutrition: intensive care. Clin Nutr 28:387-400

7. McClave SA, Lowen CC, Kleber MJ, et al (1998) Are patients fed appropriately according to their caloric requirements? J Parenter Enteral Nutr 22:375-81

8. Weir JB (1949) New methods for calculating metabolic rate with special reference to protein metabolism. J Physiol 109:1-9

9. Weekes EC (2007) Controversies in the determination of energy requirements. Proc Nutr Soc 66:367-77

10. Awad S, Stephenson MC, Placidi E, et al (2010) The effects of fasting and refeeding with a 'metabolic preconditioning' drink on substrate reserves and mononuclear cell mitochondrial function. Clin Nutr 29:538-44

11. Villet S, Chioléro RL, Bollmann MD, et al (2005) Negative impact of hypocaloric feeding and energy balance on clinical outcome in ICU patients. Clin Nutr 24:502-9
12. Dvir D, Cohen J, Singer P (2005) Computerized energy balance and complications in critically ill patients: an observational study. Clin Nutr 25:37-44

13. Arabi YM, Haddad SH, Tamim HM, et al (2010) Near-target caloric intake in critically ill medical-surgical patients is associated with adverse outcomes. JPEN J Parenter Enteral Nutr 34:280-8

14. Singer P, Anbar R, Cohen J, et al (2011) The Tight Calorie Control Study (TICACOS): a prospective, randomized, controlled pilot study of nutritional support in critically ill patients. Intensive Care Med 37:601-9

15. Casaer MP, Mesotten D, Hermans G, et al (2011) Early versus late parenteral nutrition in critically ill adults. N Engl J Med 365:506-17

16. Biolo G, Agostini F, Simunic B, et al (2008) Positive energy balance is associated with accelerated muscle atrophy and increased erythrocyte glutathione turnover during $5 \mathrm{wk}$ of bed rest. Am J Clin Nutr 88:950-8

17. Berger MM, Revelly JP, Wasserfallen JB, et al (2006) Impact of a computerized information system on quality of nutritional support in the ICU. Nutrition 22:221-9

18. Bartlett RH, Dechert RE, Mault JR, et al (1982) Measurement of metabolism in multiple organ failure. Surgery 92:771-9

19. Krishnan JA, Parce PB, Martinez S, et al (2003) Caloric intake in medical ICU patients: consistency of care with guidelines and relationship to clinical outcomes. Chest 124:297-305

20. Rubinson L, Diette GB, Song X, et al (2004) Low caloric intake is associated with nosocomial bloodstream infections in patients in the medical intensive care unit. Crit Care Med 32:350-7

21. Faisy C, Lerolle N, Dachraoui F, et al (2009) Impact of energy deficit calculated by a predictive method on outcome in medical patients requiring prolonged acute mechanical ventilation. $\mathrm{Br}$ J Nutr 101:1079-87

22. Heidegger CP, Graf S, Thibault R, et al (2011) Supplemental parenteral nutrition (SPN) in intensive care unit (ICU) patients for optimal energy coverage: improved clinical outcome. Clin Nutr (Suppl 6): in press

23. Grau T, Bonet A, Rubio M, et al (2007) Liver dysfunction associated with artificial nutrition in critically ill patients. Crit Care 11:R10

24. Herndon DN, Barrow RE, Stein M, et al (1989) Increased mortality with intravenous supplemental feeding in severely burned patients. J Burn Care Rehabil 10:309-13

25. Atkinson S, Sieffert E, Bihari D (1998) A prospective, randomized, double blind controlled clinical trial of enteral immunonutrition in the critically ill. Crit Care Med 26:1164-72

26. Battistella FD, Widergren JT, Anderson JT, et al (1997) A prospective, randomized trial of intravenous fat emulsion administration in trauma victims requiring total parenteral nutrition. J Trauma 43:52-60

27. Ahrens CL, Barletta JF, Kanji S, et al (2005) Effect of low-calorie parenteral nutrition on the incidence and severity of hyperglycemia in surgical patients: a randomized, controlled trial. Crit Care Med 33:2507-12

28. Dissanaike S, Shelton M, Warner K, O'Keefe GE (2007) The risk for bloodstream infections is associated with increased parenteral caloric intake in patients receiving parenteral nutrition. Crit Care 11:R114

29. Badjatia N, Fernandez L, Schlossberg MJ, et al (2010) Relationship between energy balance and complications after subarachnoid hemorrhage. JPEN J Parenter Enteral Nutr 34:64-9

30. Alberda C, Gramlich L, Jones N, et al (2009) The relationship between nutritional intake and clinical outcomes in critically ill patients: results of an international multicenter observational study. Intensive Care Med 35:1728-37

31. Faisy C, Llerena M, Savalle M, et al (2011) Early ICU energy deficit is a risk factor for Staphylococcus aureus ventilatorassociated pneumonia. Chest 140:1254-60 